\title{
AN ENERGY-BASED CRACK GROWTH CRITERION FOR MODELLING ELASTIC-PLASTIC DUCTILE FRACTURE
}

\author{
Zhenjun Yang \\ College of Civil Engineering and Architecture, Zhejiang University, \\ Hangzhou, 310027, P.R.China
}

ABSTRACT

A crack growth criterion is derived based on the Griffith energy concept and the cohesive zone model for modelling fracture in elastic-plastic ductile materials. The criterion is implemented in the finite element context by a virtual crack extension technique. An automatic modelling of the ductile fracture process is realised by combining a local remeshing procedure and the criterion. The validity of the derived criterion is examined by modelling a compact tension specimen.

\section{INTRODUCTION}

The crack growth in ductile elastic-plastic materials subjected to fracture has been extensively investigated to quantify the facture resistance of engineering structures. It is now widely accepted that the $J$-integral can represent the singularity of crack-tip stress and strain fields and characterize the crack initiation [1-2]. The subsequent development of $J$ resistance $\left(J_{R}\right)$ curves $(J$-integral versus crack extension) provided a crack growth criterion. However, both the $J$-integral and the $J_{R}$ curves are applicable only for small-scale yielding problems and the $J_{R}$ curves are dependent upon the geometry, size and loading conditions. The non-material nature of $J_{R}$ curves and thus nontransferability of fracture date from one specimen geometry to another, impose severe restrictions on fracture resistance prediction. The general-purposed numerical methods such as finite element (FE) method are therefore necessary in predicting large-scale yielding ductile fracture in practical engineering structures.

Two types of models have been widely used to simulate the microscopic separation mechanism in the fracture process zone (FPZ): the cohesive zone models (CZM) and crack band models (CBM). The CZMs are often implemented by nonlinear cohesive interface elements (CIEs) with zero initial thickness, whose constitutive relations are characterized by transferred stresses versus crack surface relative displacements relations (e.g., [3-6]). The CBMs model the FPZ with voidcontaining continuum cell elements with a fixed thickness, whose constitutive laws are most frequently described by the Gurson-Tvergaard (GT) model [7-8]. Most of the existing FE studies of ductile fracture, either using CZMs or CBMs, modelled Mode-I fracture in symmetric specimens subjected to symmetric loading conditions. In these cases the cracking path is a priori and thus interface elements in CZMs or cell elements in CBMs can be pre-inserted in FE meshes. The surface energy dissipation is then modelled by gradual strain softening of these elements. There is thus no need for an explicit crack propagation criterion to judge when and in which direction a crack will propagate. This, however, comes with high computational demands and restrictions in modelling practical ductile fracture problems.

In this paper, an explicit crack growth criterion for ductile fracture is derived based on the Griffith energy concept and the CZM in a view to developing an effective and efficient FE tool for modelling Mode-I and mixed-mode crack propagation in practical ductile fracture problems. A standard compact tension specimen is modelled and the numerical results are discussed.

\section{DERIVATION OF THE CRITERION}

The fundamental principle of energy conservation with respect to unit area crack extension, based on which Griffith developed the first fracture mechanics criterion, can be mathematically stated as 


$$
T_{r}=\frac{\partial}{\partial A}\left(W_{F}-U_{e}-U_{p}-E_{S}\right)\left\{\begin{array}{cc}
>0 & \text { crack propagates } \\
=0 & \text { the critical condition } \\
<0 & \text { crack does not propagate }
\end{array}\right.
$$

where $U_{e}$ is the elastic strain energy and $U_{p}$ is the plastic strain energy of the system respectively. $W_{F}$ is the work done by the externally applied loadings, $E_{s}$ is the surface dissipated energy and $A$ is the total crack surface area. The result of the first two terms in Eq. 1 is the strain energy release rate which can be regarded as a driving force of crack growth $G$

$$
G=\frac{\partial\left(W_{F}-U_{e}\right)}{\partial A}
$$

The last two terms represent the plastic energy dissipation rate and the energy dissipation rate due to crack surface separation. The summation of them represents the crack growth resistance, which is termed "energy dissipation rate" $R$ in $[3,6]$

$$
R=\frac{\partial\left(U_{p}+E_{s}\right)}{\partial A}
$$

Therefore, Eq. 1 represents when the crack driving force exceeds the crack resistance force, the crack will propagate.

A planar system subjected to surface traction vector $\mathbf{t}$ and body force vector $\mathbf{b}$ with a propagating crack is illustrated in Fig. 1. The crack is represented by the CZM characterized by the tangential traction $\sigma$-crack opening displacement (COD) relation and the shear stress $\tau$-crack sliding displacement (CSD) relation. The plastic zone shown in Fig. 1 is only for illustration.

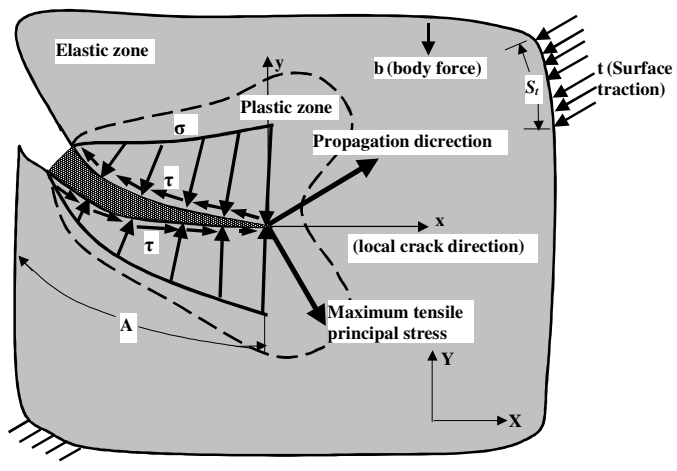

Fig. 1 A planar elastic-plastic system subjected to mixed-mode fracture
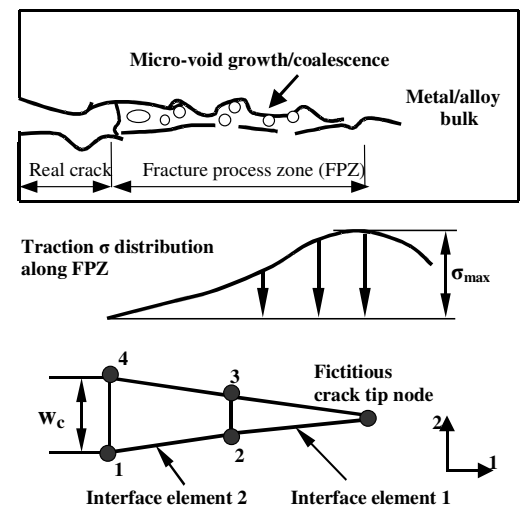

Fig. 2 Modelling of FPZ with 4-node cohesive interface elements

In the context of FE method, the external work and the total strain energy of the system $U$ can be expressed respectively as

$$
\begin{aligned}
& P=\mathbf{u}^{\mathbf{T}} \mathbf{F} \\
& U=U_{e}+U_{p}=\int_{V} W \mathrm{~d} V
\end{aligned}
$$

where $V$ is the system volume, $\mathbf{u}$ the nodal displacement vector, $\mathbf{F}$ the nodal equivalent force vector respectively and $W$ the total strain energy density. The volume integration in Eq. $3 \mathrm{~b}$ can be carried out in an element-by-element way. The strain energy of each element is calculated first using the Gauss quadrature and then a summation is carried out for all the elements as 


$$
U=\sum_{N E} \sum_{j=1}^{2} \sum_{k=1}^{2}\left\{J\left(\eta_{j}, \xi_{k}\right) \cdot W\left(\eta_{j}, \xi_{k}\right)\right\}
$$

A $2 * 2$ Gauss quadrature is used in the above equation. $N E$ is the total element number. $W\left(\eta_{j}, \xi_{k}\right)$ is the strain energy density at the Gauss point $(j, k)$ of an element where $\eta_{j}$ and $\xi_{k}$ are the isoparametric coordinates of this Gauss point. $J\left(\eta_{j}, \xi_{k}\right)$ is the product of the weighting factor and the determinant of the element Jacobian matrix at the Gauss point $(j, k)$. The strain energy density can be expressed as (the indices in the parenthesis for $J\left(\eta_{j}, \xi_{k}\right)$ and $W\left(\eta_{j}, \xi_{k}\right)$ are dropped for simplification thereafter)

$$
W=\int_{0}^{\varepsilon_{m n}} \boldsymbol{\sigma}^{T} \mathrm{~d} \boldsymbol{\varepsilon}
$$

where $\boldsymbol{\sigma}$ and $\boldsymbol{\varepsilon}$ are stress vector and strain vector at a Gauss point respectively. For a non-linear elastic material behaviour, or an elastic-plastic one under proportional loading,

$$
\frac{\partial W}{\partial A}=\frac{\partial W}{\partial \boldsymbol{\varepsilon}} \frac{\partial \boldsymbol{\varepsilon}}{\partial A}=\boldsymbol{\sigma}^{T} \frac{\partial \boldsymbol{\varepsilon}}{\partial A}
$$

Substituting Eqs. 3-6 into Eq. 1 leads to

$$
T_{r}=-\frac{\partial E_{s}}{\partial A}-\sum_{N E} \sum_{j=1}^{2} \sum_{k=1}^{2}\left\{\frac{\partial J}{\partial A} W+J \boldsymbol{\sigma}^{T} \frac{\partial \boldsymbol{\varepsilon}}{\partial A}\right\}+\frac{\partial \mathbf{u}^{T}}{\partial A} \mathbf{F}+\mathbf{u}^{T} \frac{\partial \mathbf{F}}{\partial A}
$$

Considering small deformation we have

$$
\frac{\partial \boldsymbol{\varepsilon}}{\partial A}=\frac{\partial(\mathbf{B u})}{\partial A}=\frac{\partial \mathbf{B}}{\partial A} \mathbf{u}+\mathbf{B} \frac{\partial \mathbf{u}}{\partial A}
$$

where $\mathbf{B}$ is the stain matrix. Substituting Eq. 8 into Eq. 7 and noticing that the last term of Eq. 7 is zero because the applied external loads do not change with the virtual crack extension, we have

$$
T_{r}=-\frac{\partial E_{s}}{\partial A}-\sum_{N E} \sum_{j=1}^{2} \sum_{k=1}^{2}\left\{\frac{\partial J}{\partial A} W+J \boldsymbol{\sigma}^{T} \frac{\partial \mathbf{B}}{\partial A} \mathbf{u}\right\}+\frac{\partial \mathbf{u}^{T}}{\partial A}\left[\sum_{N E} \sum_{j=1}^{2} \sum_{k=1}^{2}\left\{J \boldsymbol{\sigma}^{T} \mathbf{B}\right\}-\mathbf{F}\right]
$$

The first term in Eq. 9, i.e., the surface energy dissipation rate, can be expressed as [9]

$$
\frac{\partial E_{s}}{\partial A}=\int \frac{\partial \mathbf{d}_{\mathbf{r}}}{\partial A} \mathbf{s}_{\mathbf{t}} d A
$$

where $\mathbf{d}_{\mathbf{r}}=[\mathrm{COD}, \mathrm{CSD}]^{\mathrm{T}}$ is the relative displacement vector of the crack surface and $\mathbf{s}_{\mathbf{t}}=[\sigma, \tau]^{\mathrm{T}}$ is the transferred stress vector. Fig. 2 illustrates using two four-node CIEs to model the FPZ. These CIEs are special in that they use linear $\mathbf{d}_{\mathbf{r}}$ distribution along its length and nonlinear $\mathbf{s}_{\mathbf{t}}$ distribution [10]. Relating $\mathbf{d}_{\mathbf{r}}$ with the global nodal displacements by a linear shape function matrix $\mathbf{M}$ yields

$$
\frac{\partial E_{S}}{\partial A}=\int \frac{\partial\left(\mathbf{M}^{\mathbf{T}} \mathbf{u}\right)}{\partial A} \mathbf{s}_{\mathbf{t}} d A=\int\left[\mathbf{u} \mathbf{T} \frac{\partial \mathbf{M}}{\partial A}+\frac{\partial \mathbf{u}}{\partial A} \mathbf{T}\right] \mathbf{s}_{\mathbf{t}} d A=\mathbf{u}^{\mathbf{T}} \int \frac{\partial \mathbf{M}}{\partial A} \mathbf{s}_{\mathbf{t}} d A+\frac{\partial \mathbf{u}^{\mathbf{T}}}{\partial A} \int \mathbf{M} \mathbf{s}_{\mathbf{t}} d A
$$

Substituting Eq. 11 into Eq.9 we have

$$
T_{R}=-\sum_{N E} \sum_{j=1}^{2} \sum_{k=1}^{2}\left\{\frac{\partial J}{\partial A} W+J \boldsymbol{\sigma}^{T} \frac{\partial \mathbf{B}}{\partial A} \mathbf{u}\right\}-\mathbf{u}^{\mathbf{T}} \int \frac{\partial \mathbf{M}}{\partial A} \mathbf{s}_{\mathbf{t}} d A+\frac{\partial \mathbf{u}^{T}}{\partial A}\left[\sum_{N E} \sum_{j=1}^{2} \sum_{k=1}^{2}\left\{J \boldsymbol{\sigma}^{T} \mathbf{B}\right\}-\mathbf{F}-\int \mathbf{M} \mathbf{s}_{\mathbf{t}} d A\right]
$$

The last term in the bracket is the difference between the internal and external forces and should be zero when an equilibrium state is established. Therefore the energy criterion for ductile crack propagation becomes 


$$
-\sum_{N E} \sum_{j=1}^{2} \sum_{k=1}^{2}\left\{\frac{\partial J}{\partial A} W+J \boldsymbol{\sigma}^{T} \frac{\partial \mathbf{B}}{\partial A} \mathbf{u}\right\}-\mathbf{u}^{\mathbf{T}} \int \frac{\partial \mathbf{M}}{\partial A} \mathbf{s}_{\mathbf{t}} d A\left\{\begin{array}{lc}
>0 & \text { crack propagates } \\
=0 & \text { the critical condition } \\
<0 & \text { crack does not propagate }
\end{array}\right.
$$

The above criterion can then be calculated using a finite difference procedure with a virtual crack extension (VCE) $\triangle \mathrm{A}$. The simplest VCE is to move only the crack-tip elements so that all the non-tip elements do not contribute to Eq. 13. When this VCE and the four-node CIEs are used, Xie [10] showed that the last term in Eq. 13 could be accurately calculated by a Gauss integration scheme.

One can notice that when deriving the criterion, no assumptions are made on the fracture mode and the size of the plasticity zone. Thus the criterion is applicable for both Mode-I and mixedmode fracture problems involving either small-scale or large-scale yielding. Although Fig. 1 illustrates the planar system, the derivation for 3D problems is virtually identical except that 3-D surface CIEs should be used to model the CZM. The limitation of this criterion is that in deriving Eq. 6, a non-linear elastic material behaviour, or an elastic-plastic one under proportional loading (without unloading), must be assumed.

\section{AUTOMATIC MODELLING OF COHESIVE CRACK PROPAGATION}

It has been shown that most existing simulations of ductile cohesive crack propagation used nodal force release procedure or pre-inserted CIEs on the symmetric line and thus no mesh changing was needed during the crack propagation. In this study, a local remeshing procedure is used to automatically accommodate crack propagation. Once the criterion Eq. 13 is met, a CIE is inserted at the propagating direction. The associated $\mathrm{J}_{2}$ flow theory with an isotropic hardening is implemented within the incremental plasticity framework to model the constitutive behaviour of the bulk materials. A local normal plane arc-length constraint method [11] is used to solve the nonlinear equation system complicated by the material plasticity, material softening and continuous boundary changes. Integrated with an adaptive interface-element-number-dependent loading-step algorithm, this arc-length method has proven advantageous over other conventional solvers in modelling single and multiple cohesive crack propagation involving strong snap-back in concrete and reinforced concrete beams [12-13]. The modified Newton-Raphson iterative procedure is used.

\section{NUMEICAL EXAMPLE}

A compact tension specimen made up of AISI-1095 spheroidized steel [14] was modeled using the proposed criterion and the automatic modeling methodology. Fig. 3 shows its geometry and dimensions. Its Young's modulus, Poisson's ratio, yield strength $\sigma_{y}$, ultimate strength and ultimate strain, are $210 \mathrm{GPa}, 0.3,497 \mathrm{MPa}, 690 \mathrm{MPa}$ and 0.15 respectively. A corresponding curve with a linear hardening modulus about $1300 \mathrm{MPa}$ is used as the effective plastic strain-stress relation.

The shape and the parameters of the $\sigma$-COD relation to model the constitutive behaviour of CIEs have significant effects on FE simulations [6]. A tri-linear law shown in Fig. 4 is assumed in this study for simplicity. The critical $J$-integral to initiate a crack was found to be $65 \mathrm{~N} / \mathrm{mm}$ for the specimen [14]. This value is assumed as the area under the $\sigma$-COD relation. The following parameters are used: the cohesive strength $\sigma_{\max }=750 \mathrm{MPa}, \delta_{c}=0.116 \mathrm{~mm}$ and $\delta_{2}=0.058 \mathrm{~mm}$. $\delta_{1}$ is assumed $0.001 \mathrm{~mm}$ to give a high initial stiffness of CIEs.

Because the method of pre-inserting CIEs on the cracking line does not involve an explicit crack growth criterion, its results can be used to compare with those from the automatic modelling methodology. Fig. 5 shows a typical FE mesh with pre-inserted CIEs (MeshP). Three initial FE meshes without pre-inserted CIEs, i.e., Mesh1, Mesh2 and Mesh3 shown in Fig. 6 are designed to investigate the mesh objectivity of the proposed criterion. 


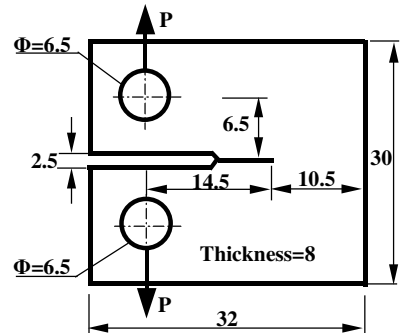

Fig. 3 Geometry and dimensions of the CT specimen (unit: $\mathrm{mm}$ )

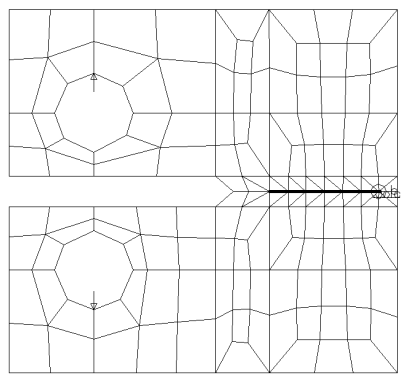

Fig. 5 FE mesh with pre-inserted cohesive interface element (MeshP)

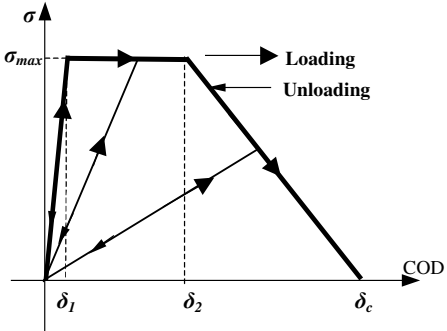

Fig. 4 COD-traction relation of cohesive interface elements

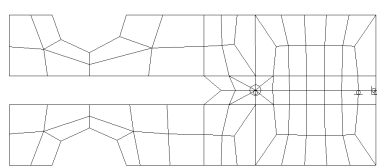

Mesh1

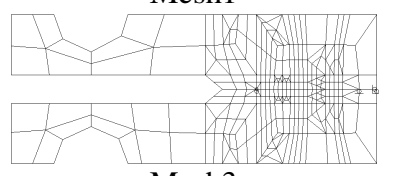

Mesh3

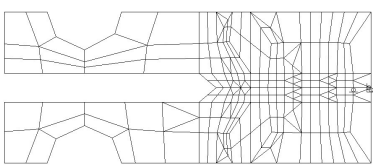

Mesh2
Fig. 6 Initial FE meshes round the cracking line used in automatic crack propagation

\section{RESULTS AND DISCUSSION}

Fig. 7 shows the evolutions of cohesive crack zone and plasticity zone simulated by the automatic modelling approach using Mesh2. In the first a few cracking steps, no plasticity occurs in the bulk material. This is perhaps because the assumed cohesive strength $\sigma_{\max }=750 \mathrm{MPa}$ is so low that the surface separation accounts for most of the dissipated energy. This is also noticed by Li and Chandra [6]. Different $\sigma$-COD relations may lead to dramatically different pictures.

Fig. 8 shows the load-load point deflection curves from the test and the finite element simulations. It can be seen that the curves from automatic modelling using the three FE meshes (Mesh1, Mesh2 and Mesh3) are almost coincident up to the peak points, which indicates the proposed criterion is mesh-objective. The response predicted by the method of pre-inserting CIEs is also very close to that by the automatic modelling approach. This demonstrates that the proposed criterion is able to correctly predict when a ductile crack propagates.

Although post-peak predictions are unreliable because unloading is not allowed when the $\mathrm{J}_{2}$ flow theory is used with the criterion, it is interesting to examine how effective the local arc-length algorithm is in passing the limiting point. Fig. 11 shows that the algorithm succeeds in passing the peak points for MeshP, Mesh1 and Mesh2 but fails for the finest mesh Mesh3. Even for the successful cases, numerical divergence occurs shortly after the peaks are reached. This inability of the current algorithm to predict post-peak responses reflects the highly nonlinearity in ductile fracture caused by material softening, large-scale plasticity and constant boundary changes. 


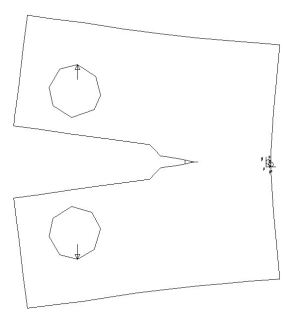

a) $\mathrm{P}=4.5 \mathrm{KN}$

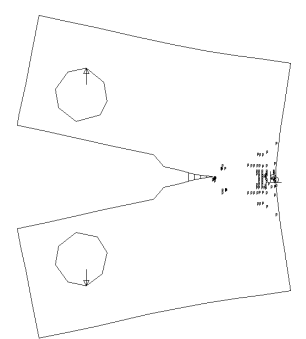

b) $\mathrm{P}=6.5 \mathrm{KN}$

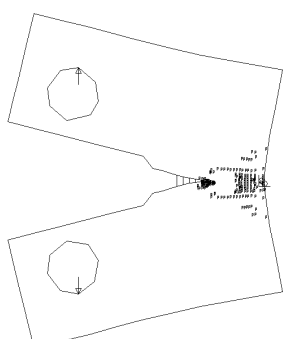

c) $\mathrm{P}=7.19 \mathrm{KN}$ (Peak load)

Fig. 7 Evolution of plasticity zone using Mesh2 (scale=50)

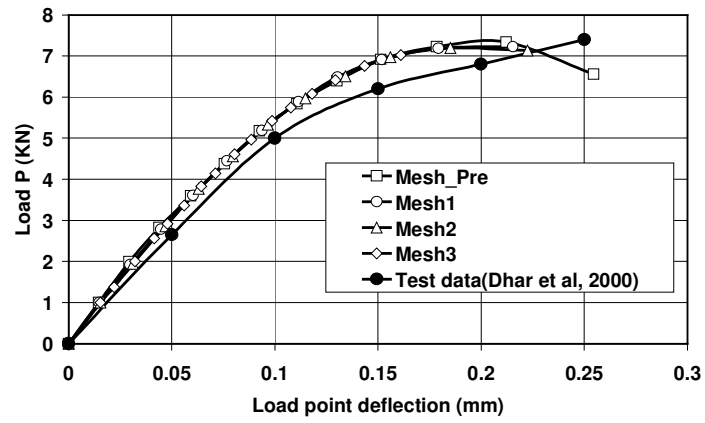

Fig. 8 Load-load point deflection curves

\section{CONCLUSION}

An explicit crack growth criterion for ductile fracture modelling has been derived based on the Griffith energy concept and the CZM. A VCE technique has been used to implement the criterion in the context of finite element method. In combination with a local remeshing procedure, an automatic modelling of the ductile fracture process has been realised. The validity and meshobjectivity of the criterion has been demonstrated by an initial numerical study of a compact tension specimen.

\section{REFERENCES}

[1] J.W. Hutchinson. J Mech Phys Solids. 16, 13 (1968).

[2] J.R. Rice, G.F. Rosengren. J Mech Phys Solids. 16, 1(1968).

[3] T. Siegmund, W. Brocks. Engng Fracture Mech. 67, 139 (2000).

[4] W.Z. Li, T. Siegmund. Engng Fracture Mech. 69, 2073 (2002).

[5] S. RoyChowdhury, S., A. Roy, R.H. Dodds. Engng Fracture Mech. 69, 983 (2002).

[6] H. Li and N. Chandra. Int J Plasticity. 19, 849 (2003).

[7] A.L. Gurson. J Engng Mater Techno. 99, 2 (1977).

[8] V. Tvergaard. Advances in Applied Mechanics. 27, 83 (1990).

[9] M. Xie, W.H. Gerstle. J Engng Mech ASCE. 121, 1349 (1995).

[10] M. Xie. Finite element modelling of discrete crack propagation. PhD thesis. University of New Mexico (1995).

[11] I.M. May, Y. Duan. Computers and Structures. 64, 297 (1997).

[12]Z.J. Yang, D. Proverbs. Engng Fracture Mech. 71, 81 (2004).

[13]Z.J. Yang, J.F. Chen. Int J Solids Struct. 41, 801 (2004).

[14] S. Dhar, P.M. Dixit, R. Sethuraman. J Press Vessels Piping. 77, 335 (2000). 